\title{
Multi Hop Secure Adhoc Network to Eradicate Cooperative Diversity
}

\author{
V. Subburaj ${ }^{12^{*}}$ and K. Chitra ${ }^{3}$ \\ ${ }^{1}$ Assistant Professor, Department of MCA,Thiagarajar School of Management, Madurai, Tamilnadu, \\ India; vsubburaj@tsm.ac.in \\ ${ }^{2}$ Ph. D Research Scholar, Manonmaniam Sundaranar University, Tirunelveli; vsubburaj@tsm.ac.in \\ ${ }^{3}$ Assistant Professor, Department of Computer Science, Government Arts College, Melur, Sivagangai Dist, \\ Tamilnadu, India; manikandan.chitra@gmail.com
}

\begin{abstract}
The concept of peer to peer mobile networks reflects on tactical network. This approach helps mobile nodes to scatter anywhere in the network and it also provide additional support for the MANET in terms of data distribution. This paper provides the result of such approach in terms of network cluster formation with secure node proximity within the boundary. This proximity paradigm will enhance the performance of network by avoid wide scattering of mobile nodes beyond the networks zone. The results shown in this paper were the work done in ns2 mobility package. The result also incorporates AODV routing protocol with IEEE 802.11 MAC for wireless network to improve network performance using mobile node proximity in conjunction with the previous work indicating the network performance degradation with these configuration. To device proximity among mobile nodes were done along with secure mechanism, such that every nodes data transmission was done only on the basis of IDS implementation with PSO fitness value. This will invoke security for every data transmission done on this network. This work has significant effect by improving network performance by $50 \%$.
\end{abstract}

Keywords: Multi Hop Cellular Network, Mobile Node Proximity, Tactical Mobile Network

\section{Introduction}

The Peer-to-Peer mobile connection is proven to be effective for data transformation between mobile nodes with a network. This lays the backbone for the every data transformation of each network irrespective of other external factors which enhance network performance. The entire performance of the network lies on the mobile nodes which provide nodes individual effective participation. If a mobile network of 1000 nodes then the network performance will be measured by the active participation of mobile nodes. This concept was addressed in Distributed Peer-to-Peer network where every nodes performance is measured which in turn provide effective data transmissions. However measuring the nodes consistency is advantageous in this form of network. To address this impact, nodes consistency were addressed using cluster layout. The work of clustered layout is to provide network performance based on homogeneous and heterogeneous network and to provide mobile node placement. Now in the case of homogeneous as well as heterogeneous node, placement may be either static or randomized. In non-homogenous network where the mobile nodes position is random, the network performance degrades as the network grows enormously.

The impact of clustering layout in mobile networking performance was not addressed properly based on the work done by Kawadia ${ }^{4}$ and Wang. Further in this

${ }^{*}$ Author for correspondence 
work the cluster layout does additional functionality of network partitioning. These works address the degradation of network based on randomized nodes sharing its part in the mobile network. The factors which affect network performance are contention, node congestion, and node depletion which were addressed in the work of Lee et al. ${ }^{6}$. These works as a whole, address the inefficiency and impact of mobile network towards clustered layout in terms of mobile node location.

Hence, we proposed mobile node proximity as an additional feature in the clustered layout as mentioned in the previous works. The impact of such approach will improve the network performance up to $50 \%$ when compared with the work of Yu et al. ${ }^{1}$. The impact features of clustered layout implements topology generation along with AODV routing algorithm modeled for internet based on the work of $\mathrm{Yu}^{1}$. We implement IEEE 802.11 for MAC protocol and also for $3 \mathrm{G}$ network which, an added feature is concerned as a part of clustered layout.

The extensive features of IEEE 802.11 along with clustered layout provide low packet delivery. These issues arose due to randomized locations of mobile nodes which were also the part of mobile network. Hence the packet delivery decreases result in low performance.

The low performance of mobile network will further affect QOS due to the lack in mobile network performance constraints. The effect of QOS was also discussed by $\mathrm{Yu}^{1}$ based on randomized Peer-to-Peer mobile network.

We also proposed the network based on Multi Hop Cellular Network instead of Multi Peer-to-Peer network since Multi Hop Cellular networks deploys single hop which connects mobile nodes with base station. In connection with the said term of Multi Hop Cellular features, which also address high frequency and larger bandwidth provides inadequate services for both voice and data centric communications. The efficient services can be retrieved from this network is by improving effective throughput and effective data delivery.

The most promising service of Multi Hop Cellular Networks are relay network where Handoff plays a vital role in mobile node service. The functionality of Handoff will be addressed in our future work after improving node performance using the concept of proximity.

The features of IDS in setting node proximity will enhance vital security mechanism in Multi Hop Cellular Network. Despite the node be randomized or scattered in terms of location, the particular node can be a part of the network using the proximity approach for effective data transformation between nodes or to the all nodes in the network. This proximity and the IDS security mechanism provide effective mobile services in terms of its QOS.

\section{Clustering Nodes based on Proximity}

By making discuss on clustering mobile nodes based on the location, our idea is to provide randomized mobile nodes approach based on RWP (Randomized Waypoint) model. The purpose of dealing with random waypoint movement is to provide random waypoint access for each and every node in MANET. Other model which was of this kind is Manhattan model.

In RWM model, all mobile nodes were deployed or fixed to a position in the network and the movements of mobile nodes were notified based on its position. Each and every mobile node position distance was measured with other nodes position. By using this measurement each and every node position were individually stored and monitored in comparison with other nodes position. This study will assist in setting up mobile node waypoint. The purpose of this waypoint is met within the closed mobile region by identifying different node position at different level in connection with other mobile nodes.

The previous work ${ }^{7}$ addresses RWP which provides connectivity and mobile node position metrics and RWP performance addressed by Mohd Izuan Mohd ${ }^{3}$. The proposed mobile node clustering mechanism based on RWP model addressed with the mobile node positioning will give mobile node positioning with the corresponding cluster.

To find mobile node proximity, first the cluster is selected based on mobile node selection and the result of this attempt would be the mobile node distance vector graph. This graph will provide mobile node association with other nodes in terms of its distance. This distance scenario will assist the network for deciding the node selection based on its proximity. This approach will enhance network by providing efficient service between nodes and better node selection based on proximity approach. To the extent such approach will also assist in efficient QOS. Figure 1 shows the mobile node position in RWP Zigzag model.

The movement of mobile nodes in this approach was retrieved using uniform velocity distribution by

$$
\mathrm{U}(0, \mathrm{~V} \max )
$$




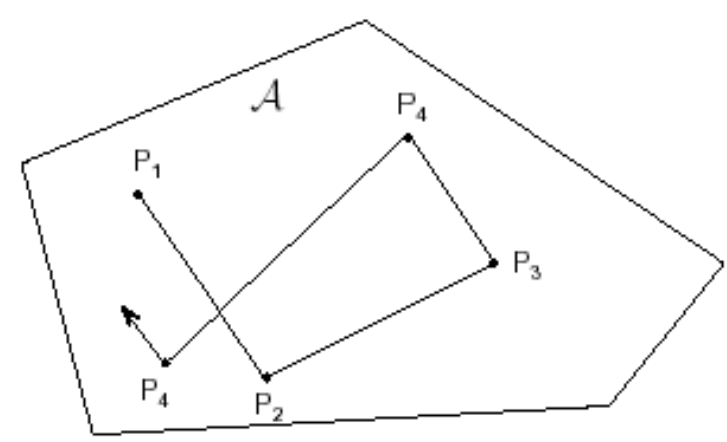

Figure 1. RWP for mobile node position.

The velocity distribution of mobile nodes is done using the formulae:

$$
\mathrm{Bi}=\sum\left(\frac{1}{\mathrm{E}}\left[\frac{1}{\mathrm{~V}}\right]\right), \mathrm{i} \neq \mathrm{j}
$$

\section{Proximity based Cluster}

\subsection{Cluster Formation}

Assume a mobile network of ten mobile nodes within a boundary. The boundary will provide the nodes extensibility in terms of the nodes waypoint movement. The mobile way point movement was addressed with the concepts of RWP ${ }^{2}$. The existing approach of RWP ${ }^{2,7}$ provides feasible mobile nodes distance based on which the mobile data transaction were done. The deputed mobile boundary was coined with the term as 'cluster'. This cluster consists of few mobile nodes along with its distance metrics. The study of RWP shows that momentum of mobile nodes will decrease and to overcome this approach the momentum was provides with uniform velocity.

The following are the sample random waypoint movement with distributed uniform approach. The simulation result were shown with $20,50,500,1000$ mobile nodes respectively. The range covered is 1.0. The RWP models were explained with Unit disk. The results shared in the Figures 2 and 3 were the work done with RWP simulator developed by us using Java Applet. This will help to find the mobile node legs and its distance as besis for our extend work. Figure 3 shows the scenario of node $\mathrm{E}$ with its neighbor node. Each node was given RWP value set with waypoint movement by giving destination nodes co-ordinates. The co-ordinates comprise of $\mathrm{x}$ and $\mathrm{y}$ variable that fix mobile node to a location in the boundary.
To finish up the way point movement ends with the parameter given in terms of speed (Node movement).

In Figures 2 and 3 the node $\mathrm{E}$ and $\mathrm{B}$ linked with its neighbor of A, B, C, D and D, C, and F respectively. The leg establishes the connection between nodes with the nearest distance measures ${ }^{8}$. Shortest the neighbor makes the node distance closer. In order to establish connection based on nearest neighbor, the leg distance is measured between the mobile nodes. If a node has more number of links, then minimum leg distance was taken into account for establishing connection and if leg distance of two or more nodes is same then the decision is taken with the assistance of RWP. Figures 4-6 show the distance measures between $\mathrm{E}$ to its corresponding neighbor making secure cluster. The number of legs found in Figures 4 is 5 in which the nearest neighbor alone considered to be in the secure cluster zone.

In Figure 6 the variable $\alpha$ measures the secure cluster zone distance and the nodes that fall within the cluster boundary are declared to be secure and the connection are establish and the variable $\beta$ is the mobile node distance to the mobile node interface boundary. The negotiation of the node $\mathrm{C}$ from the cluster boundary is based on the farthest distance from the source node and our assumption is that security breaches will intrude into the boundary based on distance measure.

Hence, the approach of cluster boundary will set the number of nodes that are legible nodes and removes the malicious node (based on distance measures) from the cluster boundary due to technical security constraints. The Figures 7-10 show the Random Waypoint Movement based on our simulation approach.

In Figure 7, enlighten dark dots were the mobile nodes and the black dots were the RWP mobile nodes. Both 


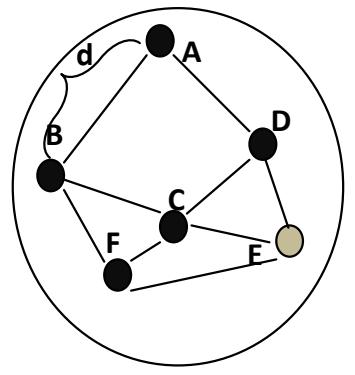

Figure 2. Scenario of node $\mathrm{B}$ and its corresponding neighbor.

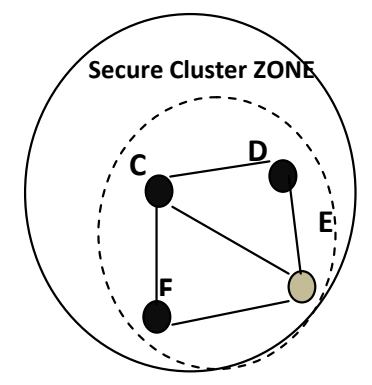

Figure 4. Scenario of node $\mathrm{E}$ and its corresponding neighbor.

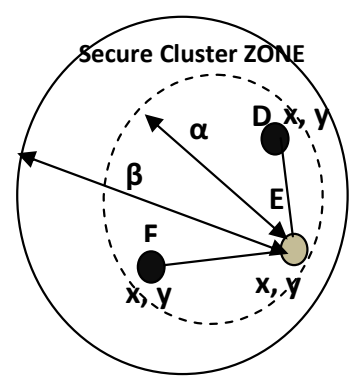

Figure 6. Neighbors in secure cluster zone.

the mobile nodes and its waypoint were put within the boundaries. In Figure 7 is the simulation result of 20 mobile nodes along with the waypoint movements and Figure 8 with 50 mobile nodes, Figure 9 with 500 and Figure 10 with 1000 mobile nodes along with its waypoint metrics.

\subsection{Secure Mobile Nodes in Cluster}

Irrespective of the size of the cluster network and no matters how big is the mobile node window size, every node in the network were deployed with IDS based Security. The understanding of this concept when a node was

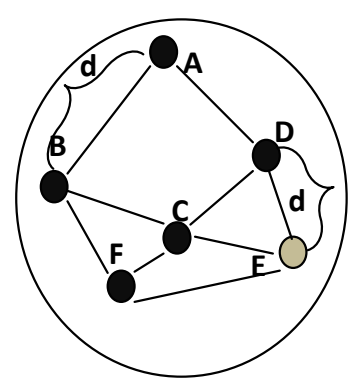

Figure 3. Scenario of node $\mathrm{E}$ and its corresponding neighbor.

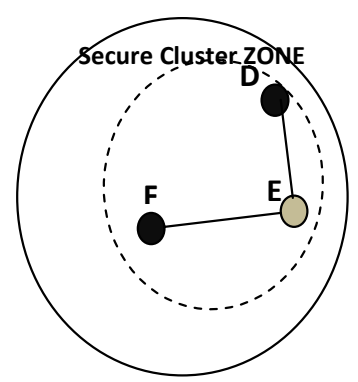

Figure 5. Neighbors in secure cluster zone.

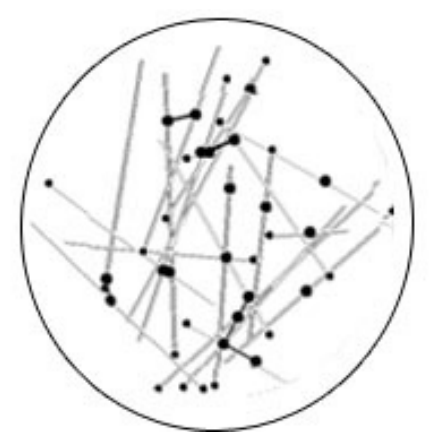

Figure 7. RWP of 20 mobile nodes with its proximity.

linked with the network based on its proximity then the nodes were deployed with IDS fitness function. The purpose is to invoke security mechanism using IDS strategy in Mobile network.

In the previous work ${ }^{9}$ we have proposed a network where mobile nodes will fight against Intrusion detection by avoiding Worm hole, black hole and web exploits etc. The fitness function used in this concept was PSO fitness function. This fitness function provides the fitness value and this value is checked for IDS consistency. In this work we compared AODV with MAODDP routing strategies for efficient routing services for setting distance measures 


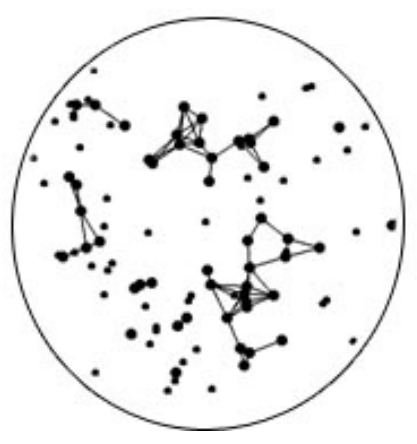

Figure 8. RWP of 50 Mobile nodes with its proximity.

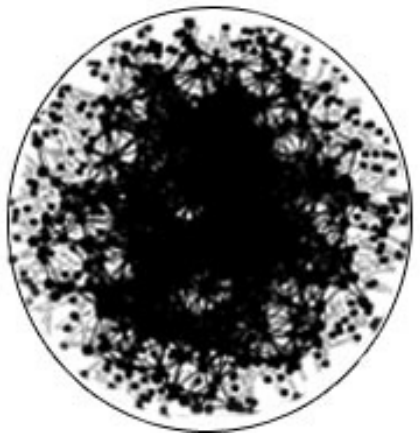

Figure 9. RWP of 500 mobile nodes with its proximity.

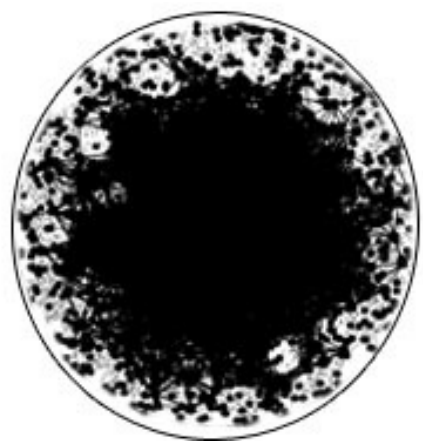

Figure 10. RWP of 1000 mobile nodes with its proximity.

from the initial node to interface boundary and distance with in cluster boundary.

We proposed the solution with AODV routing protocol for IEEE 802.11 along with mobile node proximity. The approach of waypoint makes provision for node movement and the concept of node proximity provide mobile node boundary within the proximity limit.

The proximity distances of mobile node calculate by the distance formulae.

$$
\begin{aligned}
& A=\frac{A\left(x_{3}-x_{1}\right)-B\left(x_{2}-x_{1}\right)}{2\left[\left(y_{2}-y_{1}\right)\left(x_{3}-x_{1}\right)-\left(y_{3}-y_{1}\right)\left(x_{2}-x_{1}\right)\right]} \\
& X=\frac{A-2 y\left(y_{2}-y_{1}\right)}{2\left(x_{2}-x_{1}\right)}
\end{aligned}
$$

This formulae for calculating mobile node distance was the result from a survey and to set the mobile landmark calculated by

$$
C_{i}=\frac{\sum \sqrt{\left(X_{i}-X_{j}\right)^{2}+\left(Y_{i}-Y_{j}\right)^{2}}}{\sum h_{i}}, i \neq j
$$

To set mobile node boundary from the above equation we use

$$
\mathrm{P}_{\mathrm{i}}=(\mathrm{B})^{n}=\sum_{i=1}^{n} \mathrm{~B}_{\mathrm{i}} \mathrm{C}_{\mathrm{i}}
$$

This result provides the mobile node proximity for every node within the said boundary.

To invoke the principle of IDS mechanism in every node then the proximity node will be set to act against vulnerabilities. The node within the said distance in the boundary will be consider to be a part of the network and that node will enhanced with IDS mechanism.

The IDS fitness function for each and mobile node define as

Fitness $\left(\mathrm{P}_{\mathrm{i}}\right)=\mathrm{a} / \mathrm{A}-\mathrm{b} / \mathrm{B}$

where, $\mathrm{A}$ is the cluster region, $\mathrm{B}$ is the boundary and $\mathrm{a}, \mathrm{b}$ are fitness constants.

$$
\sum_{i}^{N} C_{i} B_{i}=\sum_{i}^{N} P_{i}
$$

The above said fitness function is the result of our previous paper ${ }^{10}$ where each and every node within the boundary set with this fitness function to ensure the impact of IDS. 


\subsection{Work Flow of RWP using AODV}

Step 1: Set the boundary based on secure cluster

Step 2: Choose the mobile nodes within boundary

Step 3: Set RWP between mobile nodes

Step 4: Ensure Mobile Proximity before dynamic transaction with AODV

Step 5: Ensure IDS fitness function for all mobile nodes for security measures

\section{Simulation Result}

The Figures 11-14, graphs show the results based on RWP of mobile nodes $20,50,500$, and 1000 respectively. The result proves the fact that there is a steady growth in the RWP transmission despite the time factor is increased. In parallel with the time factor, numbers of mobile nodes were also increased and through the results it proves to

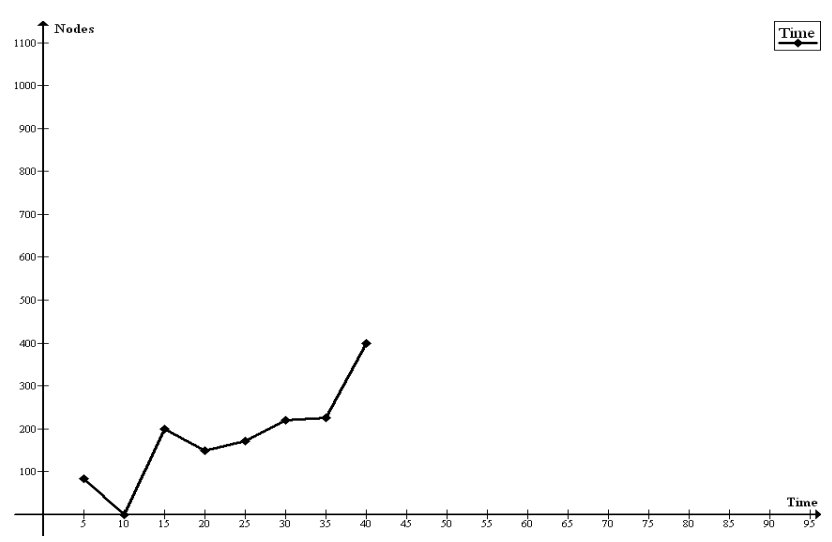

Figure 11. Node connection based on time.

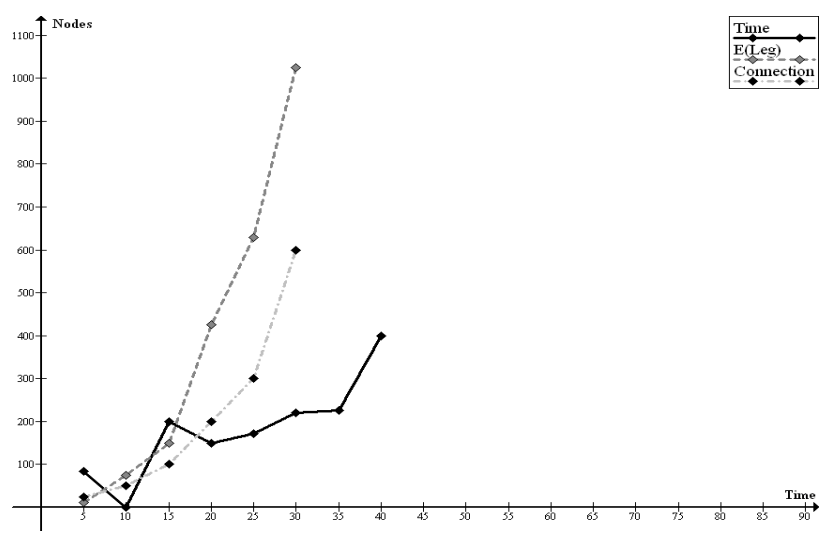

Figure 13. Node connection based on time, legs and connection. be effective based on the time and number of node sets for mobile node transaction. The attribute E (Leg) provides node limitation based on $\mathrm{E}(\mathrm{Tc})$ and $\mathrm{E}(\mathrm{Td})$ which provides proximity limitation between two nodes.

Figure 15 gives the result in terms of Time, Legs, Connections, Time with connection, and Time with Distance. Lower the value shows greater the accuracy. We have shown our result shown in the circle. Figure 16 shows the secure cluster approach with the impact of upper and lower boundary value initiatives based on mobile node cluster. The incorporated strategy shows minimized time frame par with maximized mobile nodes within the secure mobile node resides in the cluster. The proven fact of the result shows the usage of PSO optimization in secure cluster formation and avoids mobile nodes falls beyond boundary. Number of nodes falls beyond mobile boundary is 0 (zero) as per the result attained and every nodes lies within the boundary scope.

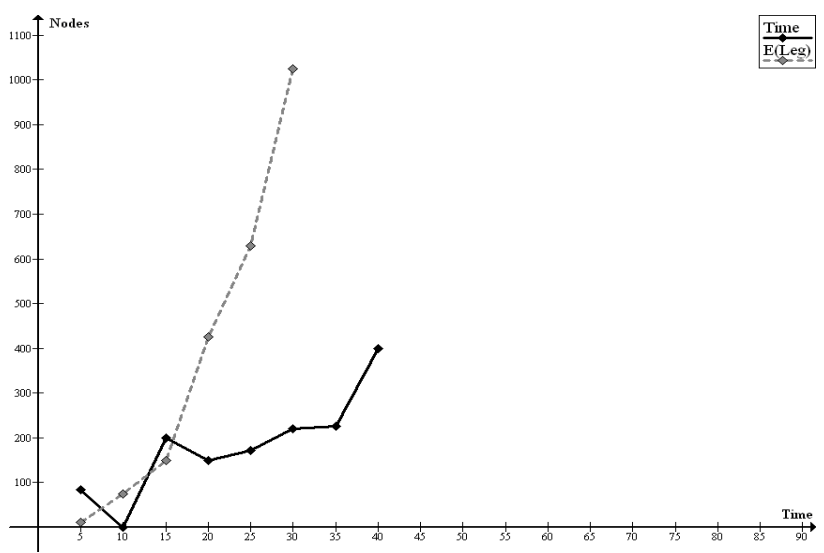

Figure 12. Node connection based on time and legs (Connection).

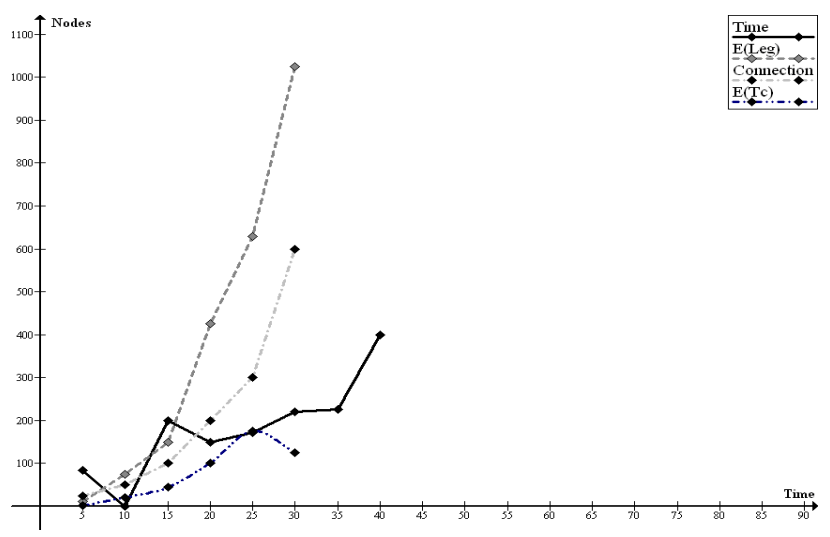

Figure 14. Node connection based on E (Tc). 


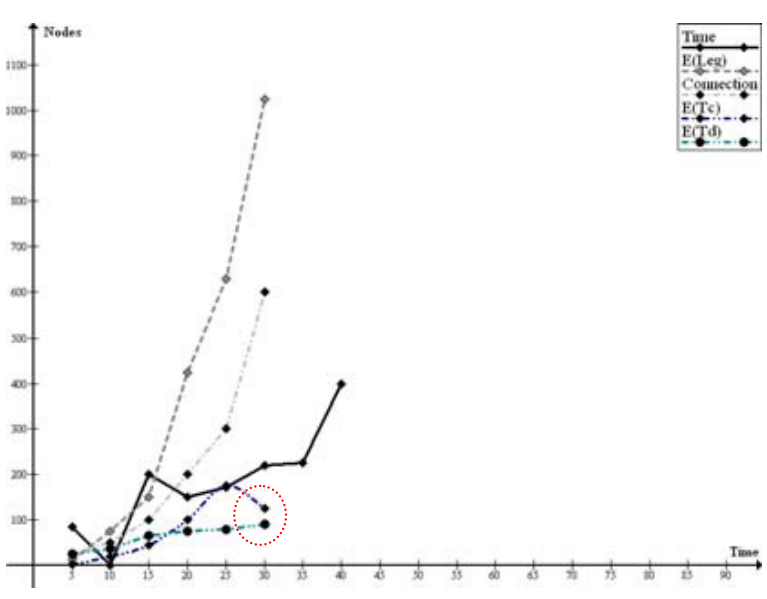

Figure 15. Node connection based on E (Td).

\section{Conclusion and Future Work}

In this work we have focused on efficient RWP along with secure mechanism using IDS for tactical mobile networks. The results received with this parameter proven to be effective based on time factor and number of mobile nodes involved in this process. The problem of RWP like slow momentum rate of mobile node velocity and Mobile Handoff mechanism will be addressed in our future works by increasing the mobile nodes size of various densities. We believe that the way the mobile node proximity was addressed in this work would help us to invoke handoff mechanism that provides better result for efficient transmission between tactical mobile networks with the optimized fitness value achieved.

\section{References}

1. Yu C, Shin KG, Lee B, Park SM, Kim H-M. Node clustering in mobile peer-to-peer multihop networks. PerCom Workshops 2006. Fourth Annual IEEE International Conference on Pervasive Computing; 2006 13-17 Mar; Pisa. p. 5-134.

2. Xiang H, Liu J, Kuang J. Minimum node degree and connectivity of two-dimensional MANETs under random waypoint mobility model. ACM Digital Library. 2010 Jun 29-Jul 1; Bradford. p. 2800-05

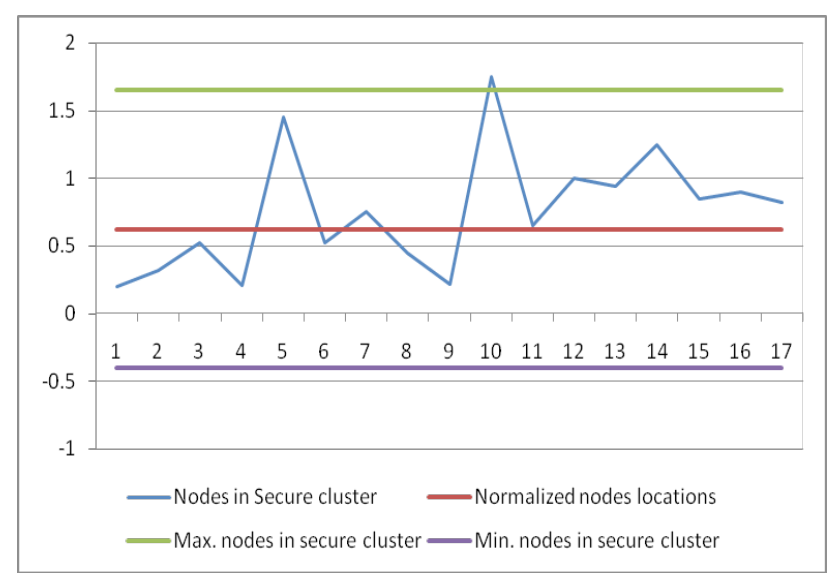

Figure 16. Secure cluster node connection based on E (Td) with optimized value.

3. Saad MIM, Zukarnain ZA. Performance analysis of random-based mobility models in MANET routing protocol. Eur J Sci Res. 2009 Jun; 32(4):444.

4. Kawadia V, Kumar PR. Power control and clustering in Ad Hoc networks. IEEE Infocom. 2003; 459-69.

5. Wang $\mathrm{KH}, \mathrm{Li}$ B. Group mobility and partition prediction in wireless Ad-Hoc networks. IEEE ICC 2002. 2002; 2:1017-21.

6. Lee S, Campbell A. HMP: hotspot mitigation protocol for mobile Ad Hoc networks. 11th IEEE/IFIP International Workshop on Quality of Service. 2003 Jun; Monterey, Canada. p. 266-86.

7. Khider I, Saad A, Furong W. Link analysis on indoor and outdoor environment for mobile Ad hoc network supported with base stations. Available from: www.mcit. gov.sa/

8. Maurya AK, Kumar A, Singh D. RWP Mobility model based performance evaluation of OLSR and LAR1 routing protocols in MANET. Int J Comput Network Comm. 2011 Nov; 3(6):145.

9. Subburaj V, Chitra K. IDS for detection and prevention of dynamic mobile node positioning based on logical distance measures. International Journal of Engineering Research and Development (IJERD). 2012 Sep; 3(7): 27-32.

10. Subburaj V, Chitra K. Mobile node dynamism using particle swarm optimization to fight against vulnerability exploitations. Int J Comput Appl Tech. 2012 Mar; 41(13):1-4. 